\title{
Teleparallel versions of Friedmann and Lewis-Papapetrou spacetimes
}

\author{
M. Sharif • M. Jamil Amir
}

Received: 2 November 2006 / Accepted: 30 January 2007 /

Published online: 20 March 2007

(C) Springer Science+Business Media, LLC 2007

\section{Erratum to: Gen Relativ Gravit 38:1735-1745 DOI 10.1007/s10714-006-0354-6}

Some minor errors have been observed which led to a change in the final result. Correcting these errors simplifies the results.

Recently, we have investigated [1] the teleparallel versions of the Friedmann models as well as the Lewis-Papapetrou solution. The tetrad and the torsion fields are obtained for both spacetimes. The tensor part of the torsion tensor of the Weitzenböck connection is given by

$$
t_{\lambda \mu \nu}=\frac{1}{2}\left(T_{\lambda \mu \nu}+T_{\mu \lambda \nu}\right)+\frac{1}{6}\left(g_{\nu \lambda} V_{\mu}+g_{\nu \mu} V_{\lambda}\right)-\frac{1}{3} g_{\lambda \mu} V_{\nu},
$$

where $\lambda, \mu, v=0,1,2,3$. When we take the variations of the indices of $t_{\lambda \mu \nu}$ in Eq. (1), there occurred some errors which affect the tensor part. If we correct these errors, the non-zero components of the tensor part, for the Friedmann models, will change to

$$
t_{010}=\frac{1}{3 f_{\kappa}(\chi)}\left\{1-f_{\kappa}^{\prime}(\chi)\right\}=t_{100},
$$

The online version of the original article can be found at http://dx.doi.org/10.1007/s10714-006-0354-6.

M. Sharif $(\varangle) \cdot$ M. Jamil Amir

Department of Mathematics, University of the Punjab,

Quaid-e-Azam Campus, Lahore 54590, Pakistan

e-mail: msharif@math.pu.edu.pk

M. Jamil Amir

e-mail: mjamil.dgk@gmail.com 


$$
\begin{aligned}
t_{001} & =-2 t_{010}, \\
t_{212} & =\frac{1}{6} a^{2} f_{\kappa}(\chi)\left\{1-f_{\kappa}^{\prime}(\chi)\right\}=t_{122}, \\
t_{221} & =-2 t_{212}, \\
t_{313} & =\frac{1}{6} a^{2} f_{\kappa}(\chi)\left\{1-f_{\kappa}^{\prime}(\chi)\right\} \sin ^{2} \theta=t_{133}, \\
t_{331} & =-2 t_{313},
\end{aligned}
$$

instead of those given by Eqs. (32)-(39) in [1].

Also, an error of minus sign has been found in Eq. (48) which affects the rest of the part. Inserting this minus sign, the corrected version of Eq. (48) is given by

$$
A^{(1)}=\frac{1}{3 h}\left\{g_{00} T_{32}^{0}+g_{02}\left(T_{32}^{2}-T_{30}^{0}\right)\right\},
$$

where $h=\sqrt{-g}=\rho \mathrm{e}^{(\gamma-\psi)}$. When we use this corrected form, the axial-vector becomes

$$
\mathbf{A}=\frac{-1}{3 \rho} \mathrm{e}^{3 \psi-\gamma}\left(\omega^{\prime} \hat{e}_{\rho}+\dot{\omega} \hat{e}_{z}\right)
$$

instead of Eq. (51) in [1]. This shows that when $\omega$ is a function only of $z$, the axial-vector will stand along the radial direction, i.e.,

$$
\mathbf{A}=\frac{-1}{3 \rho} \mathrm{e}^{3 \psi-\gamma}\left(\omega^{\prime} \hat{e}_{\rho}\right) .
$$

If $\omega$ is a function of $\rho$ only then it is given by

$$
\mathbf{A}=\frac{-1}{3 \rho} \mathrm{e}^{3 \psi-\gamma}\left(\dot{\omega} \hat{e}_{z}\right)
$$

i.e., the axial vector will lie along $z$-direction. For $\omega$ to be constant, the axialvector vanishes identically, i.e.,

$$
\mathbf{A}=0 .
$$

The spin precession of the Dirac particle in torsion gravity will also be simplified to

$$
\frac{\mathrm{d} \mathbf{S}}{\mathrm{d} t}=\frac{1}{2 \rho} \mathrm{e}^{3 \psi-\gamma}\left(\omega^{\prime} \hat{e}_{\rho}+\dot{\omega} \hat{e}_{z}\right) \times \mathbf{S}
$$

and the corresponding extra Hamiltonian will be

$$
\delta H=\frac{1}{2 \rho} \mathrm{e}^{3 \psi-\gamma}\left(\omega^{\prime} \hat{e}_{\rho}+\dot{\omega} \hat{e}_{z}\right) \cdot \sigma .
$$

\section{Reference}

1. Sharif, M., Jamil Amir, M.: Gen. Rel. Gravt. 38, 1735 (2006) 